\title{
Body Region Involvement and Quality of Life in Psoriasis: Analysis of a Randomized Controlled Trial of Adalimumab
}

\author{
April W. Armstrong ${ }^{1}$ - Delfina Guadalupe Villanueva Quintero ${ }^{2}$ Cristina M. Echeverría ${ }^{3}$. \\ Yihua $\mathrm{Gu}^{4} \cdot$ Mahinda Karunaratne ${ }^{4}$ Ofelia Reyes Servín ${ }^{4}$
}

Published online: 5 November 2016

(c) The Author(s) 2016. This article is published with open access at Springerlink.com

\begin{abstract}
Background Psoriasis severity and treatment responsiveness vary by body region, which differentially impacts quality of life (QoL).

Objective The objective of the study was to examine adalimumab efficacy by body region and regional response and QoL relationship.

Methods Patients $(n=1212)$ with moderate-to-severe psoriasis were randomized $2: 1$ to $80 \mathrm{mg}$ at week 0 , followed by adalimumab $40 \mathrm{mg}$ or placebo every other week for 16 weeks in the double-blind REVEAL study. Psoriasis Area and Severity Index (PASI) responses and Dermatology Life Quality Index outcomes were analyzed.

Results Week 16 regional mean PASI improvements were significantly greater with adalimumab $(83.1 \pm 1.57$, $81.3 \pm 1.58,75.7 \pm 1.34$, and $73.9 \pm 1.26 \%$ in the trunk, head, upper extremities, and lower extremities, respectively; all $p<0.001$ vs. placebo). Likewise, percentages of patients with regional PASI $\geq 75 / \geq 90 / 100 \%$ reduction from baseline were significantly higher with adalimumab (all $p<0.001$ );
\end{abstract}

Electronic supplementary material The online version of this article (doi:10.1007/s40257-016-0229-x) contains supplementary material, which is available to authorized users.

April W. Armstrong

april.armstrong@med.usc.edu

1 Keck School of Medicine, University of Southern California, Keith Administration Building, Room 510, 1975 Zonal Avenue, Los Angeles, CA 90089, USA

2 Instituto Dermatológico de Jalisco, Zapopan, Jalisco, Mexico

3 Department of Dermatology, Hospital Eva Perón, San Martin, Buenos Aires, Argentina

4 AbbVie Inc., North Chicago, IL, USA adalimumab responses were greater for the trunk (77.9/65.0/ $59.1 \%)$ and head (74.6/66.1/62.8\%; all $p \leq 0.0001$ vs. lower) than upper $(67.7 / 45.1 / 39.6 \% ; p=0.4, p=0.04$, $p=0.0005$, respectively, vs. lower) and lower extremities (65.7/40.0/31.3\%). Adalimumab significantly improved Dermatology Life Quality Index scores vs. placebo (8.2- vs 1.7-point decrease from baseline; $p<0.001$ ).

Limitations The study was a post hoc analysis.

Conclusions Adalimumab treatment resulted in statistically significant and clinically meaningful improvements in disease severity and QoL. QoL improvements were associated with PASI responses in all body regions.

Trial Registration ClinicalTrials.gov identifier NCT00237887.

\section{Key Points}

Psoriasis can affect a patient's quality of life, which may be related to lesion location.

Adalimumab was associated with more prominent improvements in the head and trunk regions than in the extremities.

Significant clinical improvements in regional psoriasis severity correlated with meaningful improvements in quality of life.

\section{Introduction}

Plaque psoriasis is a chronic inflammatory disease affecting approximately $3 \%$ of adults [1] that substantially impacts quality of life (QoL) [2]. Psoriasis involvement in 
distinct body regions differentially affects patient QoL. Lesions on the face and scalp have a more negative impact on QoL because of their high visibility [3, 4]. Treatment responsiveness also varies by location; areas such as the head and neck are more difficult to treat than other body regions $[3,5]$.

Treatment efficacy in clinical trials is commonly evaluated using the Psoriasis Area and Severity Index (PASI), which quantifies disease burden based on severity of erythema, desquamation, and induration, and extent of psoriatic lesions [6-8]. However, overall PASI score is weighted only by regional body surface area (BSA), failing to account for the disproportionate burden in more visible/ sensitive locations [7, 8]. The burden of psoriasis may be related to disease location and impact on QoL [8].

The relationship between treatment efficacy in different body regions and patient-reported QoL is clinically relevant, but represents a critical gap in psoriasis research. Improvements in the Dermatology Life Quality Index (DLQI) significantly correlate with PASI responses [9-12]. However, the relationship between QoL and regional disease severity has not been examined. In the REVEAL trial, the tumor necrosis factor inhibitor adalimumab significantly improved clinical disease activity vs. placebo in patients with moderate-to-severe psoriasis [13]. Using REVEAL data, we performed an exploratory analysis of regional PASI score improvements and examined their relationship with disease-related QoL.

\section{Methods}

\subsection{Study Design}

REVEAL was a 52-week, phase III, randomized study conducted in USA and Canada (NCT00237887). Patientlevel data from the 16-week, double-blind, placebo-controlled treatment period (Period A) were obtained for this analysis. Study design and patient details were previously described [13]. Briefly, patients were randomized 2:1 to receive subcutaneous adalimumab $(80 \mathrm{mg}$ at week 0 , followed by $40 \mathrm{mg}$ every other week) or placebo for 16 weeks. Enrolled patients were aged $\geq 18$ years with a clinical diagnosis of psoriasis $\geq 6$ months and stable moderate-to-severe plaque psoriasis for $\geq 2$ months before screening, defined as an affected BSA $\geq 10 \%$, PASI score $\geq 12$, and a Physician's Global Assessment of at least moderate severity at baseline [13].

\subsection{Study Assessments}

Clinical efficacy was assessed using PASI score (ranging from 0-72), a composite index based on lesion severity and percentage of BSA involved [8]. Regional PASI scores were calculated for head, trunk, upper extremities, and lower extremities as the sum of the degree of severity of erythema, induration, and desquamation $(0$, none; 1 , slight; 2 , moderate; 3 , severe; 4 , very severe), multiplied by the estimated BSA involved ( 0 , none; $1,<10 \% ; 2,10-29 \% ; 3$, 30-49\%; 4, 50-69\%; 5, 70-89\%; and 6, 90-100\%) [8]. Overall PASI score was calculated as a weighted sum of regional PASI scores based on surface area (head, 0.1; upper extremities, 0.2; trunk, 0.3; lower extremities, 0.4). Regional and overall PASI mean percentage improvements from baseline were calculated; percentages of patients achieving $\geq 75$, $\geq 90$, or $100 \%$ improvement relative to baseline (PASI 75/90/100) were determined.

Patient-reported QoL outcomes were evaluated using the DLQI questionnaire to assess the extent skin problems affect QoL. The DLQI consists of ten items answered on a scale of 0 for "not at all" to 3 for "very much" (0-30) $[8,14]$. Scores of $0 / 1$ reflect no negative effect on QoL; scores $>10$ indicate a greater negative impact on QoL. DLQI change from baseline and percentage of patients achieving a score of $0 / 1$ were calculated.

\subsection{Statistical Analysis}

Analyses were performed on the intent-to-treat population from Period A, defined as all patients randomized at baseline. Percentage improvement and PASI 75/90/100 responses were determined for patients with non-zero baseline values. Mean percentage changes from baseline in PASI and DLQI were compared using one-way analysis of variance. Differences in PASI responses were compared between treatment groups using Fisher's exact test. Correlation between change from baseline PASI score and DLQI score at week 16 was analyzed using a Pearson correlation coefficient. Missing data were imputed using last observation carried forward for continuous variables or non-responder imputation for categorical variables.

\section{Results}

\subsection{Patients}

As previously reported, demographics and baseline disease characteristics for the 1212 patients enrolled in REVEAL were balanced across adalimumab $(n=814)$ and placebo $(n=398)$ groups [13] (Table 1). Overall, 52.6\% of patients had moderate psoriasis, and $47.4 \%$ had severe/very severe psoriasis. QoL was greatly affected; mean DLQI score was 11.4. Mean overall PASI score was 18.9; regional PASI scores were higher for lower (21.5) and upper (18.2) extremities compared with the head (16.8) and 
trunk (16.7). During Period A, 31 patients (3.8\%) discontinued adalimumab; $43(10.8 \%)$ discontinued placebo.

\subsection{Efficacy Assessments}

\subsubsection{Clinical Efficacy}

Mean overall and regional PASI scores for patients receiving adalimumab were significantly improved at all weeks compared with patients receiving placebo ( $p<0.001$; Fig. 1a). The majority of improvements in regional PASI scores for patients receiving adalimumab were achieved by week 4 . At week 16, mean percentage improvements in regional PASI scores with adalimumab were numerically higher in the trunk $(83.1 \%)$ and head
(81.3\%), followed by the upper $(75.7 \%)$ and lower extremities $(73.9 \%)$. Mean percentage improvements in regional PASI scores were significantly higher for the trunk compared with the upper and lower extremities $(p<0.0001)$ and for the head compared with the upper $(p=0.0019)$ and lower $(p<0.0001)$ extremities.

Compared with placebo, adalimumab provided significantly higher PASI 75/90/100 responses across all body regions at every visit $(p<0.001$ except PASI 100 in lower extremities at week $4 ; p=0.03$; Fig. 1b). Responses were more rapid and substantial in the head and trunk than in the upper and lower extremities. At week 16, PASI 75/90/100 responses in patients receiving adalimumab were numerically higher in the trunk $(77.9 / 65.0 / 59.1 \%$, respectively) and head $(74.6 / 66.1 / 62.8 \%)$ than upper $(67.7 / 45.1 / 39.6 \%)$
Table 1 Baseline characteristics of patients with psoriasis [13]

\begin{tabular}{|c|c|c|}
\hline Characteristic & Placebo $(n=398)$ & Adalimumab $(n=814)$ \\
\hline Mean \pm SD age (years) & $45.4 \pm 13.4$ & $44.1 \pm 13.2$ \\
\hline Male, $n(\%)$ & $257(64.6)$ & $546(67.1)$ \\
\hline White, $n(\%)$ & $359(90.2)$ & $742(91.2)$ \\
\hline Mean \pm SD weight $(\mathrm{kg})$ & $94.1 \pm 23.0$ & $92.3 \pm 23.0$ \\
\hline Mean \pm SD BMI $\left(\mathrm{kg} / \mathrm{m}^{2}\right)$ & $31.6 \pm 7.5$ & $30.9 \pm 7.1$ \\
\hline Tobacco user, $n(\%)$ & $136(34.2)$ & $274(33.7)$ \\
\hline Alcohol user, $n(\%)$ & $273(68.6)$ & $567(69.7)$ \\
\hline Mean \pm SD psoriasis duration (years) & $18.4 \pm 11.9$ & $18.1 \pm 11.9$ \\
\hline PsA history, $n(\%)$ & $113(28.4)$ & $224(27.5)$ \\
\hline Mean \pm SD BSA affected $(\%)$ & $25.6 \pm 14.8$ & $25.8 \pm 15.5$ \\
\hline \multicolumn{3}{|l|}{ Mean \pm SD PASI score } \\
\hline Overall & $18.8 \pm 7.1$ & $19.0 \pm 7.1$ \\
\hline Head & $17.0 \pm 11.6$ & $16.7 \pm 10.7$ \\
\hline Upper extremities & $17.9 \pm 8.3$ & $18.4 \pm 8.3$ \\
\hline Trunk & $16.8 \pm 9.6$ & $16.7 \pm 10.0$ \\
\hline Lower extremities & $21.2 \pm 9.3$ & $21.6 \pm 9.1$ \\
\hline Mean \pm SD DLQI score & $11.4 \pm 7.0$ & $11.4 \pm 6.6$ \\
\hline \multicolumn{3}{|l|}{ PGA score, $n(\%)$} \\
\hline Moderate & $220(55.3)$ & $417(51.2)$ \\
\hline Severe & $155(38.9)$ & $346(42.5)$ \\
\hline Very severe & $23(5.8)$ & $51(6.3)$ \\
\hline \multicolumn{3}{|l|}{ Previous psoriasis treatment ${ }^{\mathrm{a}}, n(\%)$} \\
\hline Topical therapy & $290(72.9)$ & $618(75.9)$ \\
\hline Phototherapy & $59(14.8)$ & $138(17.0)$ \\
\hline Systemic nonbiologic & $88(22.1)$ & $188(23.1)$ \\
\hline Systemic biologic & $53(13.3)$ & 97 (11.9) \\
\hline Laser & 0 & $1(0.1)$ \\
\hline Mean CRP $(\mathrm{mg} / \mathrm{L})^{\mathrm{b}}$ & 7.3 & 8.0 \\
\hline
\end{tabular}

$B M I$ body mass index, $B S A$ body surface area, $C R P$ C-reactive protein, $D L Q I$ Dermatology Life Quality Index, PASI Psoriasis Area and Severity Index, PGA Physician's Global Assessment (for psoriasis), PsA psoriatic arthritis, $S D$ standard deviation

a Within 12 months of study treatment

b Placebo, $n=394$; adalimumab, $n=808$ 
a

Overall

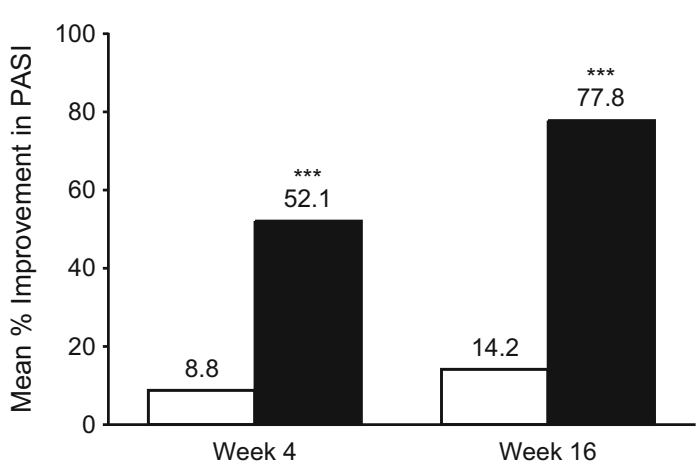

Placebo

Adalimumab

Head

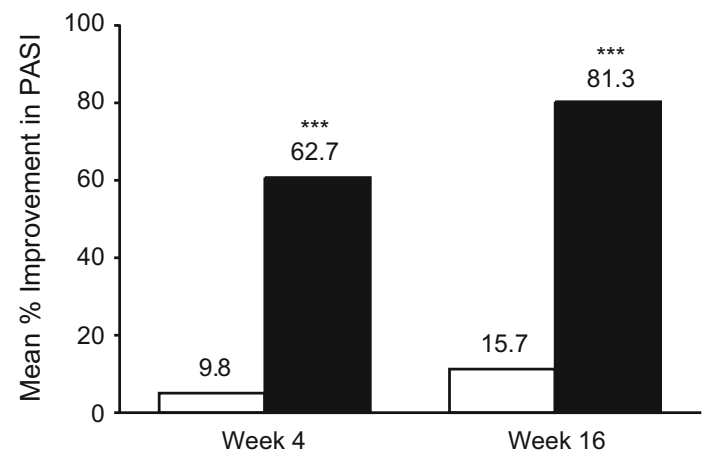

Upper Extremities

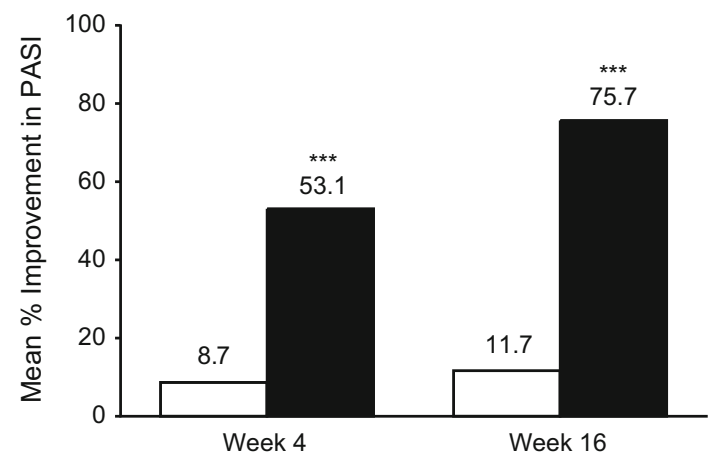

Fig. 1 a Mean percentage improvement from baseline in PASI scores through week 16 overall and by region (head, trunk, upper extremities, and lower extremities) for the ITT population (LOCF). b Proportion of patients achieving PASI 75, 90, and 100 responses through week 16 overall and by region (head, trunk, upper

and lower extremities (65.7/40.0/31.3\%). Compared with lower extremities, the head and trunk showed significant improvements in PASI 75/90/100 response rates $(p \leq 0.0001)$. Although the difference between the upper and lower extremities was not significant for PASI 75 $(p=0.4)$, the differences were significant for PASI 90 and 100 ( $p=0.04$ and $p=0.0005$, respectively).

Regional PASI 75/90/100 responses at week 16 were analyzed by baseline overall PASI scores $(\leq 20,>20$ to
Trunk

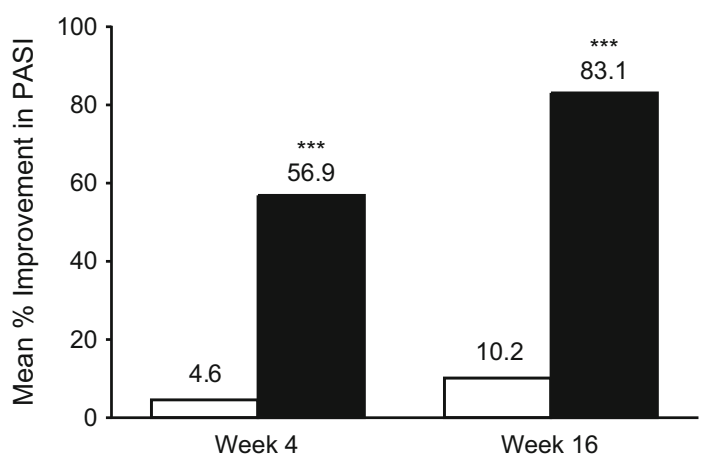

\section{Lower Extremities}

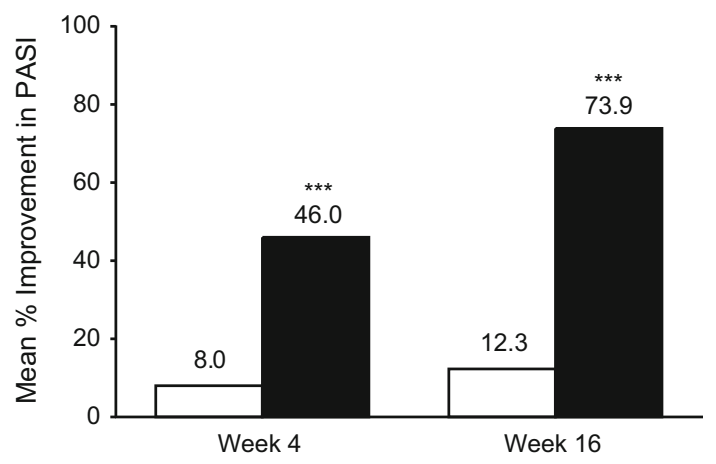

extremities, and lower extremities) in the ITT population (nonresponder imputation). ADA adalimumab, ITT intent-to-treat, $L O C F$ last observation carried forward, PASI Psoriasis Area and Severity Index, $P B O$ placebo, $* * * p<0.001, * p<0.05$, adalimumab vs. placebo group

$\leq 30,>30$; Fig. 2). Overall and regional PASI response rates were significantly higher with adalimumab vs. placebo. Regional PASI response rates with adalimumab were generally higher in patients with a lower baseline PASI regional score. PASI 100 response rates were numerically higher for the head and trunk, regardless of baseline PASI scores or treatment. For patients with a regional baseline (a composite of the baseline scores of the head/trunk/upper extremities/lower extremities) PASI score $>30$, PASI 100 
b

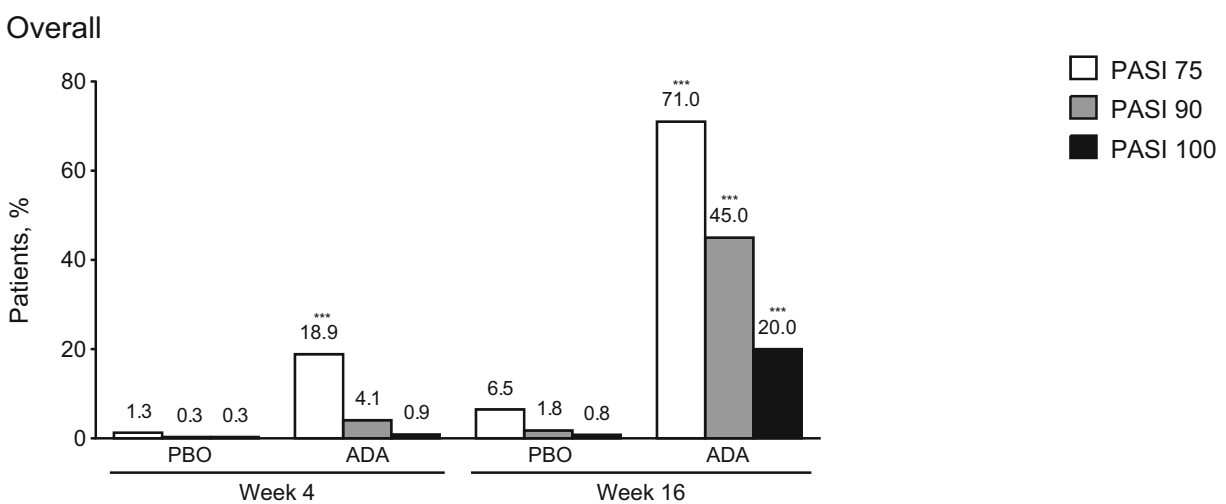

Head

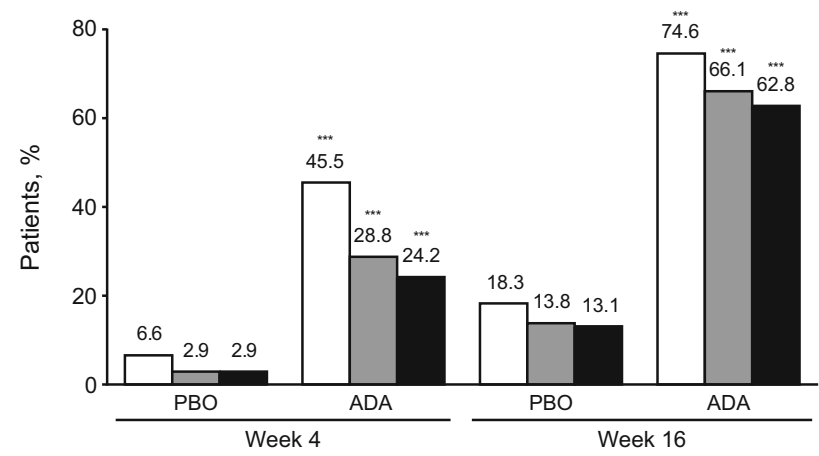

Upper Extremities

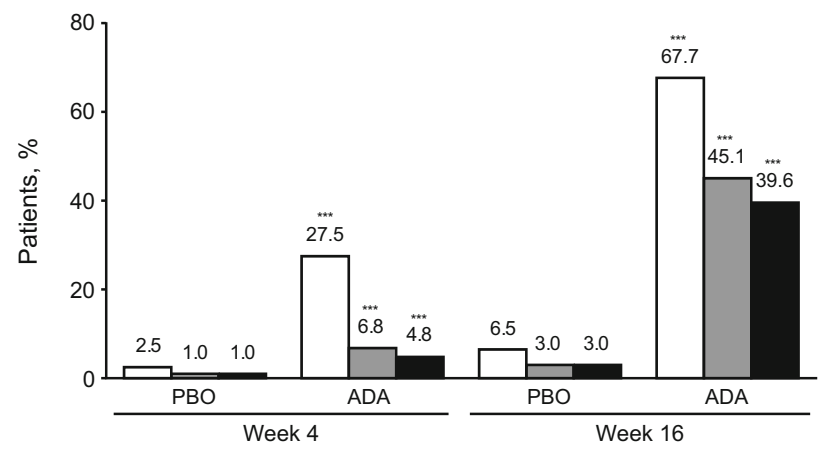

Fig. 1 continued

response rates were $40.9 / 48.6 / 31.3 / 27.4 \%$ for the head/ trunk/upper extremities/lower extremities with adalimumab vs. 0/0/0/4.4\% with placebo, indicating no spontaneous total clearance in PASI.

\subsubsection{Quality of Life}

Significantly greater DLQI improvements were observed in patients receiving adalimumab vs. patients receiving placebo at weeks 4 and $16(p<0.001$; Fig. 3), with 6.3- and 8.2-point reductions (improvements), respectively, in mean DLQI scores, vs. a 1.7-point reduction at both weeks for placebo-treated patients.

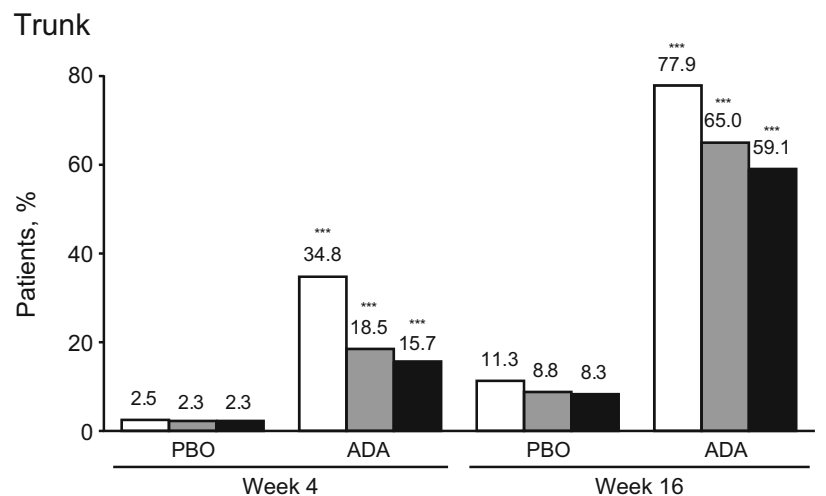

Lower Extremities

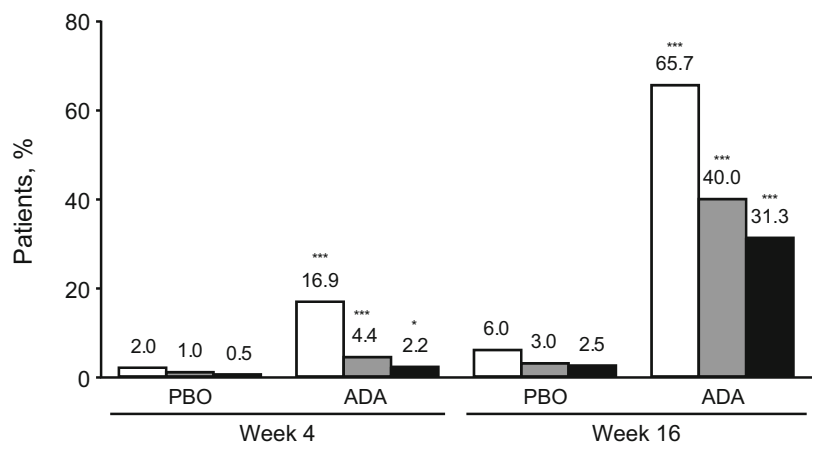

\subsubsection{Association Between Regional PASI and DLQI Scores}

Mean percentage change from baseline in DLQI and proportion of patients achieving DLQI scores of $0 / 1$ by levels of PASI reduction are presented in Fig. 4. At the threshold of PASI 75 to $<90$ response for individual regions, the mean percentage change in DLQI was numerically lower in the head $(-59.6 \%)$ vs. the trunk $(-71.9 \%)$ and upper ($78.2 \%)$ and lower extremities (-78.4\%; Fig. 4a). In patients achieving an overall PASI 100 response, there was a mean $-93.5 \%$ change in DLQI; regional PASI 100 response rates for the head/trunk/upper extremities, and 

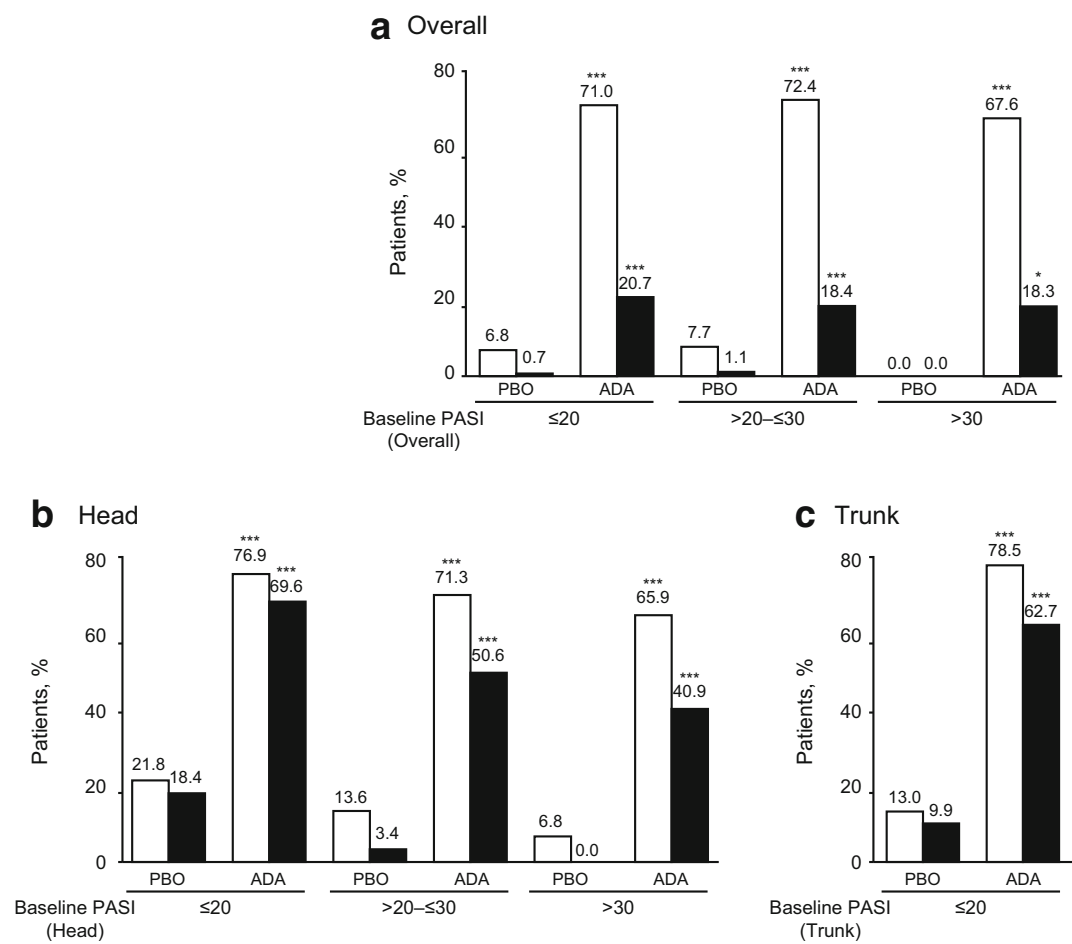

$$
\text { C Trunk }
$$

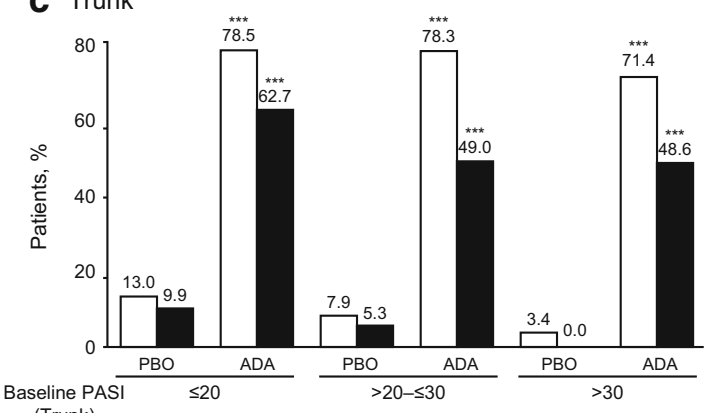

d Upper Extremities

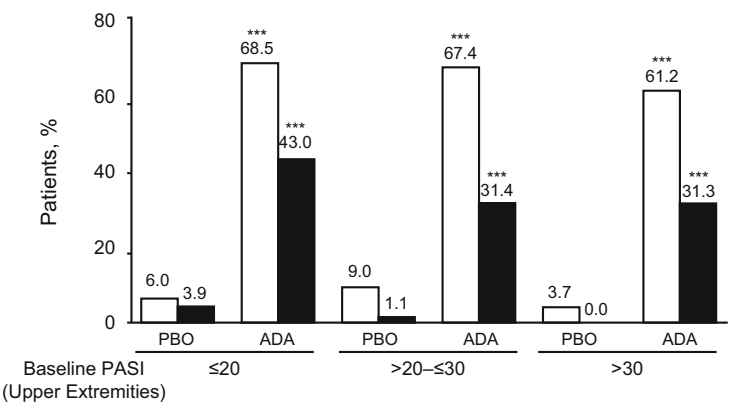

e Lower Extremities

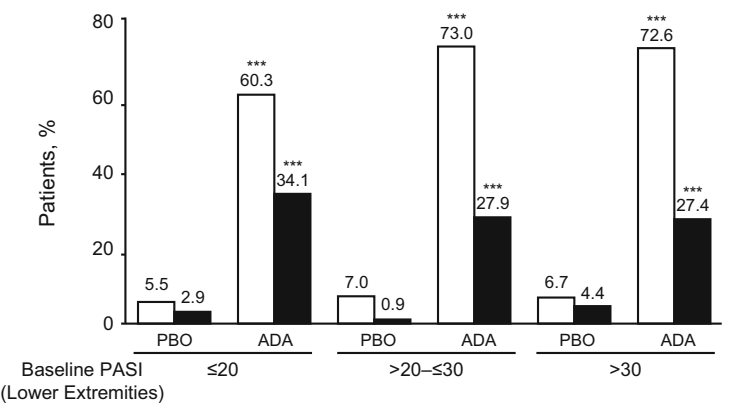

Fig. 2 Proportion of patients achieving PASI 75 and 100 responses at week 16 by baseline PASI scores in a overall, $\mathbf{b}$ head, $\mathbf{c}$ trunk, $\mathbf{d}$ upper extremities, and e lower extremities (ITT population; nonresponder imputation). Baseline PASI categories correspond to the overall PASI

score (a) or the regional PASI score (b-e); includes patients with nonzero baseline PASI scores in the respective region. ADA adalimumab, ITT intent-to-treat, PASI Psoriasis Area and Severity Index, $P B O$ placebo, $* * * p \leq 0.001 ; * p<0.05$, adalimumab vs. placebo group

Fig. 3 Mean \pm SE change from baseline in DLQI at weeks 4 and 16 in the ITT population (LOCF). Scores range from 0 to 30 , with higher scores indicating increasing impact on quality of life. DLQI Dermatology Life Quality Index, ITT intent-totreat, $L O C F$ last observation carried forward, $S E$ standard error, $* * * p<0.001$

adalimumab vs. placebo group

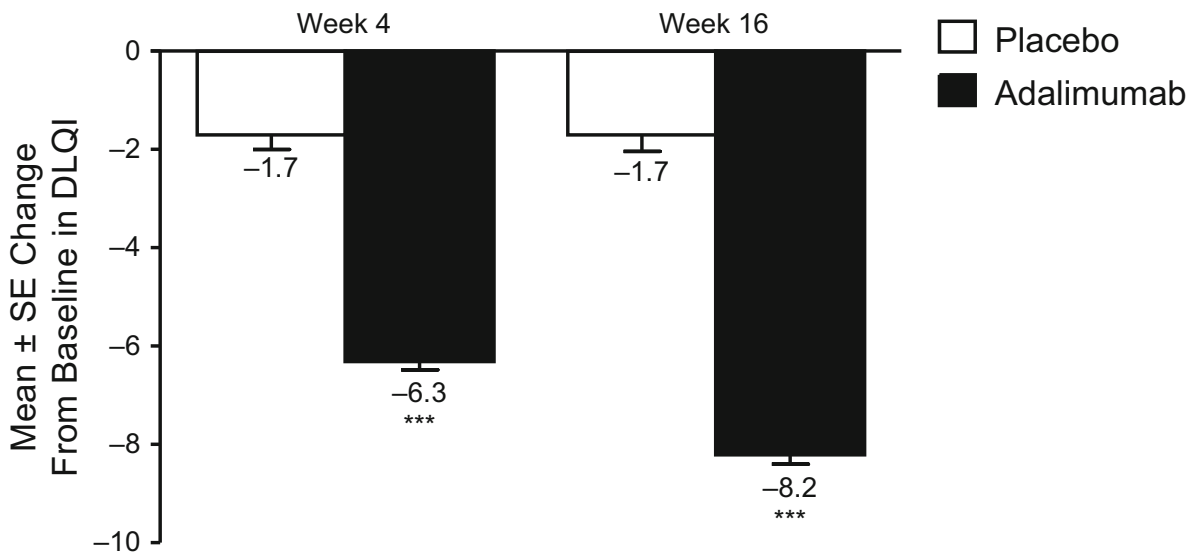


Fig. 4 a Mean percentage improvements from baseline in DLQI scores. b Proportion of patients with a DLQI score of 0 or 1 at week 16 by regional PASI response in the ITT population (LOCF). DLQI Dermatology Life Quality Index, ITT intent-to-treat, $L O C F$ last observation carried forward, $P A S I$ Psoriasis Area and Severity Index

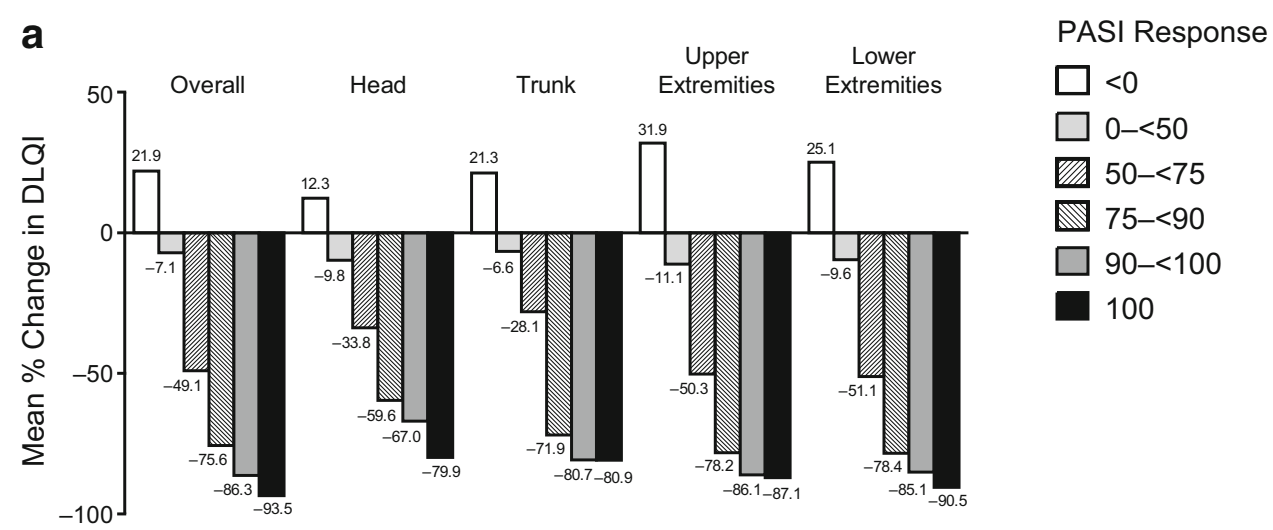

b

PASI Response

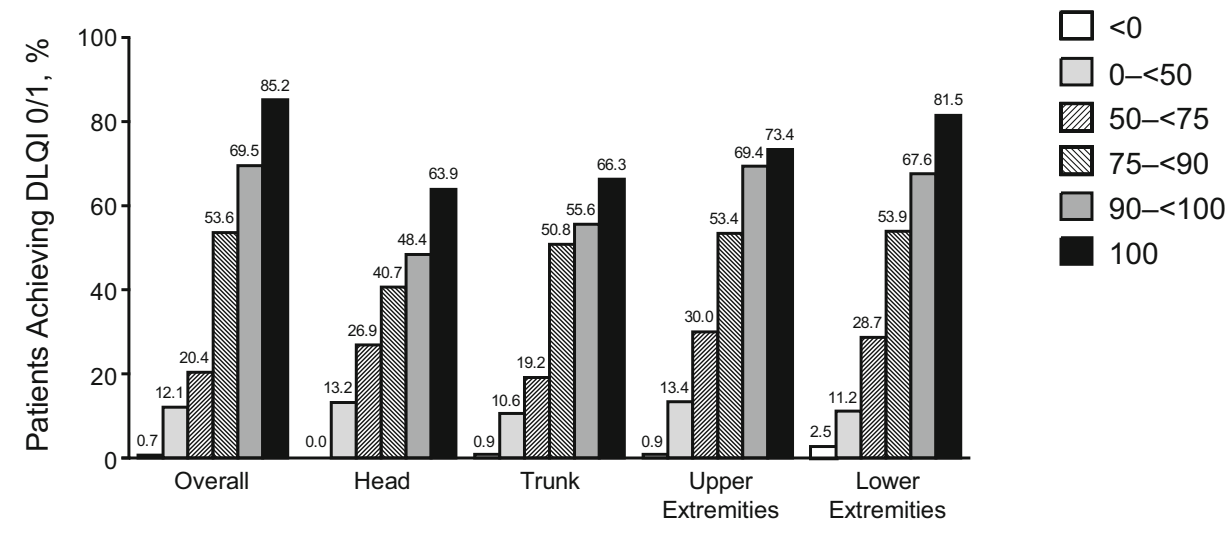

lower extremities were associated with mean percentage changes in DLQI of $-79.9 /-80.9 /-87.1$, and $-90.5 \%$, respectively. Among patients who achieved DLQI scores of $0 / 1$ and a threshold PASI score of 75/90/100 for a body region, a minority had PASI scores that failed to meet the same threshold in the three remaining body regions (Supplemental Table 1).

Achieving a DLQI score of $0 / 1$ after 16 weeks of treatment with adalimumab was associated with improvements in overall and regional PASI scores (Fig. 4b). The correlation between change from baseline in PASI score and change from baseline in DLQI score at week 16 was significant for the overall PASI score and for all body regions $(p<0.001)$. Among patients with an overall PASI 75 to $<90$ response, $53.6 \%$ achieved a DLQI score of 0/1. Regional PASI 75 to $<90$ responses yielded similar DLQI 0/1 responses (50.8-53.9\%), except for the head (40.7\%). Of patients with an overall PASI 100 response, $85.2 \%$ achieved a DLQI score of 0/1. Additionally, DLQI 0/1 scores were associated with regional PASI 100 responses of $63.9 / 66.3 / 73.4$, and $81.5 \%$ for the head/trunk/upper extremities, and lower extremities. For the head, trunk, and lower extremities, achieving a PASI 100 vs. a PASI $<100$ response had a major impact on the percentage of patients achieving a DLQI score of 0/1. The distribution of change in DLQI score by PASI response categories for each body region is in Supplemental Fig. 1.

\subsection{Safety Assessments}

As reported previously [13], adverse events were typically mild to moderate and more frequent in patients receiving adalimumab than placebo (62.2 vs. $55.5 \%)$. Serious adverse events occurred in $2 \%$ of patients in either group. Serious infections occurred in 0.6 and $1.0 \%$ of patients receiving adalimumab and placebo respectively. No patients had an adverse event of tuberculosis in the doubleblind period. Two patients in the adalimumab group and one in the placebo group had malignancies other than nonmelanoma skin cancer.

\section{Discussion}

Understanding the impact of regional psoriasis severity on QoL and its response to therapeutics is critical for identifying therapies that maximize clinical outcomes and QoL. Overall, these results showed that adalimumab provided significant clinical improvements across body regions and was associated with improved QoL. Clinical improvements 
occurred rapidly (significant PASI improvements occurred by week 4 across all regions) and regional PASI scores improved significantly through 16 weeks of treatment. Improvements in PASI scores were faster and more prominent in the head and trunk than in the extremities. Notably, the greatest response at week 4 occurred in the difficult-to-treat head region [3]. These findings are important because the emotional and psychosocial effects on QoL caused by lesion visibility are significant.

For patients, both symptom improvement and alleviation of QoL impairments are important. PASI scores do not always correspond with the impact psoriasis has on QoL $[15,16]$. Based on the mean baseline DLQI score, psoriasis had a "very large effect" on QoL in the REVEAL population [17]. The mean DLQI improvement at week 16 was similar to a REVEAL study subanalysis (range 7.1-9.6; mean 8.3) [18]. Furthermore, rapid and significant improvements in DLQI were achieved with adalimumab by week 4, and scores continued to improve through week 16. Our results are clinically meaningful, as the difference between adalimumab and placebo groups met or exceeded the 2.3- to 5.7-point between-group difference that is suggested to be clinically relevant [10].

Both the mean percentage improvement in DLQI scores and the proportion of patients achieving a DLQI score of $0 / 1$ at week 16 were associated with regional PASI responses. These results are consistent with previous studies demonstrating an association between overall improvements in PASI and DLQI with adalimumab $[10,11,19]$. A recent systematic review of randomized controlled trials with biologics used to treat psoriasis demonstrated a significant correlation between mean reductions in overall PASI and DLQI $(r=0.898$; $p<0.01$ ) [20]. However, changes were not assessed by body region. Our findings indicate that the degree in which clinical improvements were associated with QoL varied by body region. Although the head and trunk were more responsive to adalimumab, there was a higher threshold for PASI responses in these regions to significantly impact DLQI scores. Additionally, despite the head and trunk achieving higher PASI 75/90/100 rates, a lower percentage of patients with improvements in the head and trunk achieved a DLQI score of $0 / 1$. This could be because other body areas may still have residual psoriasis.

The relationship between regional PASI improvement and DLQI improvement is consistent with the previous observation that psoriasis treatment goals are rarely met without achieving at least a PASI 75 response [21], and demonstrates that the degree to which alleviation of the clinical symptoms of psoriasis improves QoL depends on which area is affected. A PASI 75 response has predominated as the primary endpoint in clinical studies, yet a recent opinion has suggested PASI 90 response as a more relevant measure with regard to QoL [22]. In our analysis, incremental achievement in PASI response was generally associated with incremental DLQI 0/1 achievement. When comparing PASI 90 vs. PASI 75 responders for the head and trunk, the incremental benefit of achieving PASI 90 response was limited. However, approximately half of patients achieving PASI 75 response and the majority achieving PASI 100 response for each body region achieved a DLQI score of $0 / 1$, indicating little or no remaining impact on their QoL.

A limitation of this study was that the methods used to score PASI responses did not distinguish the specific distribution of improvements within each body region, such as difficult-to-treat areas (e.g., scalp) or visible areas (e.g., face and hands). Additionally, there is no QoL instrument that can be used to study PASI improvement by specific body region. Finally, this was a post hoc analysis.

\section{Conclusions}

Treatment with adalimumab for 16 weeks provided statistically and clinically significant improvements in PASI responses across body regions, as well as in DLQI scores. Improvements in QoL corresponded with PASI responses in each region, with a lower threshold for PASI responses in the extremities to impart an improvement in QoL. Conversely, there was a higher threshold for treatment response in the head before QoL improved, underscoring the need for effective therapy to clear psoriatic lesions in this highly visible region.

Acknowledgements Editorial and medical writing support was provided by Katherine Groschwitz, $\mathrm{PhD}$, at Complete Publication Solutions, LLC. This support was funded by AbbVie Inc.

\section{Compliance with Ethical Standards}

Funding AbbVie Inc. funded the study (NCT00237887), contributed to its design, and was involved in the collection, analysis, and interpretation of the data, and in the writing, review, and approval of the publication.

Conflict of interest April W. Armstrong serves as an investigator and/or consultant for AbbVie Inc., Amgen, Janssen, Merck, Lilly, Celgene, Novartis, Pfizer, and Modernizing Medicine. Delfina Guadalupe Villanueva Quintero has served as a consultant and speaker for UCB Pharma, Leo Pharma, Pfizer, and AbbVie Inc. Cristina M. Echeverría has served as a consultant and speaker for AbbVie Inc. and UCB Pharma. Yihua Gu, Mahinda Karunaratne, and Ofelia Reyes Servín are employees of AbbVie Inc. and may own AbbVie Inc. stock and/or stock options.

Ethics approval and consent to participate This is a post hoc analysis of a previously published study (Menter et al. Adalimumab therapy for moderate-to-severe psoriasis: a randomized, controlled phase III trial. J Am Acad Dermatol. 2008;58:106-115). The original study was conducted in accordance with the protocol, International 
Conference on Harmonisation guidelines, applicable regulations and guidelines governing clinical study conduct, and the ethical principles that have their origin in the 1964 Helsinki Declaration and its later amendments.

Written informed consent for the original study was obtained from all individual participants.

Open Access This article is distributed under the terms of the Creative Commons Attribution-NonCommercial 4.0 International License (http://creativecommons.org/licenses/by-nc/4.0/), which permits any noncommercial use, distribution, and reproduction in any medium, provided you give appropriate credit to the original author(s) and the source, provide a link to the Creative Commons license, and indicate if changes were made.

\section{References}

1. Parisi R, Symmons DP, Griffiths CE, Ashcroft DM. Global epidemiology of psoriasis: a systematic review of incidence and prevalence. J Investig Dermatol. 2013;133(2):377-85.

2. Rapp SR, Feldman SR, Exum ML, et al. Psoriasis causes as much disability as other major medical diseases. J Am Acad Dermatol. 1999;41(3 Pt 1):401-7.

3. van de Kerkhof PC, Murphy GM, Austad J, et al. Psoriasis of the face and flexures. J Dermatol Treat. 2007;18(6):351-60.

4. Kragballe K, Menter A, Lebwohl M, et al. Long-term management of scalp psoriasis: perspectives from the International Psoriasis Council. J Dermatol Treat. 2013;24(3):188-92.

5. Wozel G. Psoriasis treatment in difficult locations: scalp, nails, and intertriginous areas. Clin Dermatol. 2008;26(5):448-59.

6. Puzenat E, Bronsard V, Prey S, et al. What are the best outcome measures for assessing plaque psoriasis severity? A systematic review of the literature. J Eur Acad Dermatol Venereol. 2010;24(Suppl 2):10-6.

7. Robinson A, Kardos M, Kimball AB. Physician Global Assessment (PGA) and Psoriasis Area and Severity Index (PASI): why do both? A systematic analysis of randomized controlled trials of biologic agents for moderate to severe plaque psoriasis. J Am Acad Dermatol. 2012;66(3):369-75.

8. Mease PJ. Measures of psoriatic arthritis: Tender and Swollen Joint Assessment, Psoriasis Area and Severity Index (PASI), Nail Psoriasis Severity Index (NAPSI), Modified Nail Psoriasis Severity Index (mNAPSI), Mander/Newcastle Enthesitis Index (MEI), Leeds Enthesitis Index (LEI), Spondyloarthritis Research Consortium of Canada (SPARCC), Maastricht Ankylosing Spondylitis Enthesis Score (MASES), Leeds Dactylitis Index (LDI), Patient Global for Psoriatic Arthritis, Dermatology Life Quality Index (DLQI), Psoriatic Arthritis Quality of Life (PsAQOL), Functional Assessment of Chronic Illness Therapy-Fatigue (FACIT-F), Psoriatic Arthritis Response Criteria (PsARC), Psoriatic Arthritis Joint Activity Index (PsAJAI), Disease Activity in Psoriatic Arthritis (DAPSA), and Composite Psoriatic
Disease Activity Index (CPDAI). Arthritis Care Res. 2011;63(Suppl 11):S64-85.

9. Shikiar R, Bresnahan BW, Stone SP, et al. Validity and reliability of patient reported outcomes used in psoriasis: results from two randomized clinical trials. Health Qual Life Outcomes. 2003;1:53.

10. Shikiar R, Willian MK, Okun MM, et al. The validity and responsiveness of three quality of life measures in the assessment of psoriasis patients: results of a phase II study. Health Qual Life Outcomes. 2006;4:71.

11. Revicki DA, Willian MK, Menter A, et al. Relationship between clinical response to therapy and health-related quality of life outcomes in patients with moderate to severe plaque psoriasis. Dermatology. 2008;216(3):260-70.

12. Feldman SR, Menter A, Koo JY. Improved health-related quality of life following a randomized controlled trial of alefacept treatment in patients with chronic plaque psoriasis. Br J Dermatol. 2004;150(2):317-26.

13. Menter A, Tyring SK, Gordon K, et al. Adalimumab therapy for moderate to severe psoriasis: a randomized, controlled phase III trial. J Am Acad Dermatol. 2008;58(1):106-15.

14. Mrowietz U, Kragballe K, Reich K, et al. Definition of treatment goals for moderate to severe psoriasis: a European consensus. Arch Dermatol Res. 2011;303(1):1-10.

15. Kirby B, Richards HL, Woo P, et al. Physical and psychologic measures are necessary to assess overall psoriasis severity. J Am Acad Dermatol. 2001;45(1):72-6.

16. Weiss SC, Kimball AB, Liewehr DJ, et al. Quantifying the harmful effect of psoriasis on health-related quality of life. J Am Acad Dermatol. 2002;47(4):512-8.

17. Hongbo Y, Thomas CL, Harrison MA, et al. Translating the science of quality of life into practice: what do Dermatology Life Quality Index scores mean? J Investig Dermatol. 2005;125(4):659-64.

18. Kimball AB, Bensimon AG, Guerin A, et al. Efficacy and safety of adalimumab among patients with moderate to severe psoriasis with co-morbidities: subanalysis of results from a randomized, double-blind, placebo-controlled, phase III trial. Am J Clin Dermatol. 2011;12(1):51-62.

19. Revicki DA, Willian MK, Menter A, et al. Impact of adalimumab treatment on patient-reported outcomes: results from a phase III clinical trial in patients with moderate to severe plaque psoriasis. J Dermatol Treat. 2007;18(6):341-50.

20. Mattei PL, Corey KC, Kimball AB. Psoriasis Area Severity Index (PASI) and the Dermatology Life Quality Index (DLQI): the correlation between disease severity and psychological burden in patients treated with biological therapies. J Eur Acad Dermatol Venereol. 2014;28(3):333-7.

21. Mrowietz U, Kragballe K, Reich K, et al. An assessment of adalimumab efficacy in three phase III clinical trials using the European Consensus Programme criteria for psoriasis treatment goals. Br J Dermatol. 2013;168(2):374-80.

22. Puig L. PASI90 response: the new standard in therapeutic efficacy for psoriasis. J Eur Acad Dermatol Venereol. 2015;29(4):645-8. 